\title{
NUMBER VERSUS SIZE
}

\author{
STEPHEN WATSON
}

(Communicated by Dennis Burke)

\begin{abstract}
We show, for example, that normal collectionwise Hausdorff spaces which are collectionwise normal with respect to families of cardinality $\boldsymbol{N}_{1}$ are collectionwise normal with respect to any number of copies of $[0,1]$.

We also show that normal collectionwise Hausdorff spaces are collectionwise normal with respect to scattered paracompact sets.
\end{abstract}

This paper examines several aspects of the question: When is a normal collectionwise Hausdorff space collectionwise normal with respect to discrete families of closed sets with some property? We show that a sufficient condition is collectionwise normality with respect to families of a fixed cardinality. For example, normal collectionwise Hausdorff spaces which are collectionwise normal with respect to families of cardinality $\aleph_{1}$ must be collectionwise normal with respect to any number of copies of $[0,1]$. Estimates are provided which depend on either the hereditary Lindelöf number or the Lindelöf number and cardinality of the underlying sets being separated. We show that another sufficient condition is that the underlying sets being separated be scattered and paracompact. The first result shows that the construction of normal collectionwise Hausdorff spaces which are collectionwise normal with respect to families of cardinality less than $\kappa$ but not collectionwise normal with respect to families of cardinality $\kappa$ essentially requires that the sets which cannot be separated be big. The second result contrasts with Fleissner's normal collectionwise Hausdorff space of 1973 [1] which is not collectionwise normal with respect to scattered sets $\left(\omega_{1}\right)$ and Navy's normal collectionwise Hausdorff space of 1979 [2] which is not collectionwise normal with respect to paracompact sets $\left(\left(D\left(\omega_{1}\right)\right)^{\omega}\right)$.

We begin with a general result.

THEOREM 1. If $X$ is a normal collectionwise Hausdorff space and discrete families of cardinality at most $\lambda+$ are separated, then discrete families of closed paracompact sets of hereditary Lindelöf degree at most $\lambda$ are separated.

ProOF. Let $\left(A_{\alpha}: \alpha \in \kappa\right)$ be a discrete family of nonempty closed paracompact subsets of $X$ of hereditary Lindelöf degree at most $\lambda$. Let $A=\bigcup\left\{A_{\alpha}: \alpha \in \kappa\right) . A$ is a paracompact subspace of $X$. By the collectionwise Hausdorff property, we can define discrete families of open sets in $X,\left\{U_{\alpha \beta}: \alpha \in \kappa\right\}$, inductively on $\beta \in \lambda^{+}$

Received by the editors November 8, 1985 and, in revised form, December 5, 1986.

1980 Mathematics Subject Classification (1985 Revision). Primary 54D15; Secondary 54A25, 54 G20.

This paper has been supported by the Natural Sciences and Engineering Research Council of Canada. 
such that

1. $\bigcup\left\{U_{\alpha \gamma}: \gamma<\beta\right\}$ either contains $A_{\alpha}$ or does not contain $U_{\alpha \beta} \cap A_{\alpha}$.

2. $\overline{U_{\alpha \beta}} \cap A \subset A_{\alpha}$.

This is possible, since if we have defined $\left\{U_{\alpha \gamma}: \alpha \in \kappa\right\}$ for each $\gamma<\beta$, we may choose $\left\{P_{\alpha}: \alpha \in \kappa\right\}$ such that $p_{\alpha}$ is an element of $A_{\alpha}-\bigcup\left\{U_{\alpha \gamma}: \gamma<\beta\right\}$ whenever $A_{\alpha}-\bigcup\left\{U_{\alpha \gamma}: \gamma<\beta\right\}$ is nonempty and $p_{\alpha}$ is an element of $A_{\alpha}$ otherwise. Let $\left\{U_{\alpha \beta}: \alpha \in \kappa\right\}$ be a discrete family of open sets separating $\left\{P_{\alpha}: \alpha \in \kappa\right\}$ such that $\overline{U_{\alpha \beta}} \cap A \subset A_{\alpha} . \quad p_{\alpha} \in U_{\alpha \beta} \cap A_{\alpha}$ implies that $\bigcup\left\{U_{\alpha \gamma}: \gamma<\beta\right\}$ does not contain $U_{\alpha \beta} \cap A_{\alpha}$ unless $\bigcup\left\{U_{\alpha \gamma}: \gamma<\beta\right\}$ contains $A_{\alpha}$ as required. For each $\beta \in \lambda^{+}$, let $U_{\beta}=\bigcup\left\{U_{\alpha \beta}: \alpha \in \kappa\right\} \cap A .\left\{U_{\beta}: \beta \in \lambda^{+}\right\}$is an open cover in the subspace $A$. This is true, since, if $\left\{U_{\beta}: \beta \in \lambda^{+}\right\}$is not an open cover in $A$, then there is $\alpha \in \kappa$ such that $\bigcup\left\{U_{\beta}: \beta \in \lambda^{+}\right\}$does not contain $A_{\alpha}$, thus, such that $\bigcup\left\{U_{\alpha \beta}: \alpha \in \kappa, \beta \in \lambda^{+}\right\}$ does not contain $A_{\alpha}$ and thus (since $U_{\gamma \beta} \cap A_{\alpha}=\varnothing$ whenever $\gamma \neq \alpha$ ) such that $\bigcup\left\{U_{\alpha \beta}: \beta \in \lambda^{+}\right\}$does not contain $A_{\alpha}$. In this case, for each $\beta \in \lambda^{+}, \bigcup\left\{U_{\alpha \gamma}: \gamma \in\right.$ $\beta\}$ does not contain $A_{\alpha}$ and so $\bigcup\left\{U_{\alpha \gamma}: \gamma \in \beta\right\}$ does not contain $U_{\alpha \beta} \cap A_{\alpha}$. This implies that $\left\{\bigcup\left\{U_{\alpha \gamma}: \gamma \in \beta\right\} \cap A_{\alpha}: \beta \in \lambda^{+}\right\}$is a strictly increasing sequence of open subsets of $A_{\alpha}$ which contradicts the assumption that the hereditary Lindelöf degree of $A_{\alpha}$ is at most $\lambda$. Let $\left\{V_{\beta, n}: \beta \in \lambda^{+}, n \in \omega\right\}$ be an open refinement of $\left\{U_{\beta}: \beta \in \lambda^{+}\right\}$in $A$ such that, for each $n \in \omega,\left\{V_{\beta, n}: \beta \in \lambda^{+}\right\}$is a discrete family and such that, for each $n \in \omega$ and $\beta \in \lambda^{+}, \overline{V_{\beta, n}} \subset U_{\beta}$. (This is possible, since $A$ is paracompact and since $V_{\beta, n}=\bigcup\left\{V_{i, n}: V_{i, n} \subset U_{\beta}\right\}$, if $\left\{V_{i, n}: n \in \omega, i \in I_{n}\right\}$ is an open refinement of $\left\{U_{\beta}: \beta \in \lambda^{+}\right\}$in $A$ such that, for each $n \in \omega$ and $i \in I_{n}$, $\overline{V_{i, n}} \subset U_{\beta}$ and $\left\{V_{i, n}: i \in I_{n}\right\}$ is a discrete family.) $\left\{\overline{V_{\beta, n}}: \beta \in \lambda^{+}\right\}$is a discrete family of closed sets in $X$. Let $\left\{W_{\beta, n}: \beta \in \lambda^{+}\right\}$be a discrete family of open sets such that $W_{\beta, n} \supset \overline{V_{\beta, n}}$ and such that $W_{\beta, n} \cap A \subset U_{\beta} \cap A$. Let $G_{n}=\left\{W_{\beta, n} \cap U_{\alpha, \beta}: \beta \in\right.$ $\left.\lambda^{+}, \alpha \in \kappa\right\}$ for each $n \in \omega$. Each $G_{n}$ is a discrete family of open sets whose closures intersect at most one $A_{\alpha}$ (since $\overline{W_{\beta, n} \cap U_{\alpha, \beta}} \cap A \subset \overline{U_{\alpha, \beta}} \cap A \subset A_{\alpha}$ ) and $\bigcup\left\{G_{n}: n \in\right.$ $\omega\}$ covers $A$ (since $\bigcup\left\{W_{\beta, n}: \beta \in \lambda^{+}, n \in \omega\right\} \supset \bigcup\left\{V_{\beta, n}: \beta \in \lambda^{+}, n \in \omega\right\} \supset A$ and $\left.\bigcup\left\{U_{\alpha, \beta}: \alpha \in \kappa\right\} \supset U_{\beta} \supset U_{\beta} \cap A \supset W_{\beta, n} \cap A\right)$. The proof is complete.

This theorem has a simpler statement for metric sets.

COROLLARY 1. If $X$ is a normal collectionwise Hausdorff space and discrete families of cardinality at most $\lambda^{+}$are separated, then discrete families of closed metric sets of weight at most $\lambda$ are separated.

Additional estimates are provided by

THEOREM 2. Let $X$ be a normal collectionwise Hausdorff space.

(i) If discrete families of cardinality at most $\lambda^{\aleph_{0}}$ are separated, then discrete families of closed Lindelöf sets of cardinality at most $\lambda$ are separated.

(ii) If discrete families of cardinality at most $\lambda$ are separated, then discrete families of compact sets of cardinality at most $\lambda$ are separated. (This theorem is implied by Theorem 1 and Theorem 3 when the sets are metric.)

PROOF. Let $\left\{A_{\alpha}: \alpha<\kappa\right\}$ be a discrete family of closed Lindelöf (compact) sets of cardinality at most $\lambda$. For each $\alpha<\kappa$, let $\left\{b_{\alpha}^{\gamma}: \gamma \in \lambda\right\}$ list $A_{\alpha}$. For each $\gamma \in \lambda$, let $\left\{U_{\alpha}^{\gamma}: \alpha \in \kappa\right\}$ be a discrete family of open sets such that

1. $\overline{U_{\alpha}^{\gamma}} \cap A_{\beta}=\varnothing(\beta \neq \alpha)$,

2. $b_{\alpha}^{\gamma} \in U_{\alpha}^{\gamma}$. 
For each $\alpha \in \kappa$, let $f(\alpha) \in[\lambda]^{\omega}\left([\lambda]^{<\omega}\right)$ such that $\bigcup\left\{U_{\alpha}^{\gamma}: \gamma \in f(\alpha)\right\} \supset A_{\alpha}$. $|\mathrm{rng} f| \leq \lambda^{\omega}(\lambda)$ and so, since discrete families of cardinality at most $\lambda^{\omega}(\lambda)$ are separated, we may assume, without loss of generality, that there is $A \in[\lambda]^{\omega}\left([\lambda]^{<\omega}\right)$ such that, for each $\alpha \in \kappa, \bigcup\left\{U_{\alpha}^{\gamma}: \gamma \in A\right\} \supset A_{\alpha} .\left\{U_{\alpha}^{\gamma}: \gamma \in A, \alpha \in \kappa\right\}$ is a $\sigma$-discrete family of open sets, the closure of each element of which intersects at most one $A_{\alpha}$, and the proof is complete.

The proof of Theorem 2 has been simplified by a comment of Ken Kunen.

Next, we prove

THEOREM 3. If $X$ is a normal collectionwise Hausdorff space, then discrete families of closed paracompact scattered sets are separated.

We need two preliminary lemmas, the first of which is due to Telgarsky [3].

LEMMA 1. Scattered paracompact spaces are ultraparacompact (that is, any open cover has a disjoint open refinement).

LEMMA 2. Scattered paracompact spaces are the free union of subspaces of successor rank.

ProOF. Let $X$ be a counterexample of minimal rank $\alpha . \alpha$ is a limit ordinal, and so a disjoint open refinement of an open cover of subspaces of rank less than $\alpha$ is a decomposition of $X$ as the free union of subspaces of rank less than $\alpha$, each of which, by minimality, is the free union of subspaces of successor rank.

PROOF OF THEOREM 3. Let $\left(X_{\alpha}: \alpha \in I\right)$ be a discrete family of closed paracompact scattered sets. We construct $\left(X_{\alpha}^{i}, B_{\alpha}^{i}, D_{\alpha}^{i}, U_{\alpha}^{i}: \alpha \in I, i \in \omega\right)$ by

1. $X_{\alpha}^{0}=X_{\alpha}$.

2. $B_{\alpha}^{i}$ is such that

(i) $\bigoplus B_{\alpha}^{i}=X_{\alpha}^{i}$,

(ii) each element of $B_{\alpha}^{i}$ has successor rank,

(iii) $B_{\alpha}^{i}$ refines $B_{\alpha}^{j}$ whenever $j<i$.

(This is possible by Lemma 2 and because the free union of free unions is a free union.)

3. $D_{\alpha}^{i}$ is the set of points of maximum rank in some element of $B_{\alpha}^{i}$.

4. $U_{\alpha}^{i}$ is a discrete family of open sets whose closures intersect at most one $X_{\alpha}$ such that $U_{\alpha}^{i} \supset D_{\alpha}^{i}$ (this is possible since $U\left\{D_{\alpha}^{i}: \alpha \in I\right\}$ is a discrete family of points).

5. $X_{\alpha}^{i+1}=X_{\alpha}^{i}-\bigcup U_{\alpha}^{i}$.

If we can show that for each $\alpha \in I$ there is $i \in \omega$ such that $X_{\alpha}^{i}=\varnothing$ then we are finished. Suppose not and let $x \in X_{\alpha}-\bigcup U_{\alpha}^{i}$ for each $i \in \omega$. By induction, we can find, for each $i \in \omega, B_{i} \in B_{\alpha}^{i}$ such that $x \in B_{i}$. For each $i \in \omega, B_{i+1}$ is contained in $B_{i}$ minus the points of maximum rank and so $\operatorname{rk}\left(B_{i+1}\right)<\operatorname{rk}\left(B_{i}\right)$ whenever $B_{i}$ is nonempty. Each $B_{i}$ is nonempty and so $\left\{\operatorname{rk}\left(B_{i}\right): i \in \omega\right\}$ is a descending sequence of ordinals.

The rationals are not scattered but

THEOREM 4. Normal collectionwise Hausdorff spaces are collectionwise normal with respect to countable sets. 
PROOF. If $\left\{A_{\alpha}: \alpha \in \kappa\right\}$ is a discrete family of countable sets and $\left\{x_{\alpha}^{n}: n \in \omega\right\}$ lists $A_{\alpha}$, let $\left\{U_{\alpha}^{n}: \alpha \in \kappa\right\}$ be a discrete family of open sets such that $x_{\alpha}^{n} \in U_{\alpha}^{n}$ and $\overline{U_{\alpha}^{n}} \cap A_{\beta}=\varnothing(\beta \neq \alpha)$. $\left\{U_{\alpha}^{n}: \alpha \in \kappa, n \in \omega\right\}$ is a $\sigma$-discrete family of open sets whose closures intersect at most one $A_{\alpha}$ and which cover the $A_{\alpha}$ 's. By the standard subtraction argument, $\left\{A_{\alpha}: \alpha \in \kappa\right\}$ is separated.

We conclude with:

PROBLEM. Characterize those spaces $Y$ for which there exists a normal collectionwise Hausdorff space which is not collectionwise normal with respect to copies of $Y$.

Many partial results on this problem are stated in [4].

\section{BIBLIOGRAPHY}

1. W. G. Fleissner, A normal, collectionwise Hausdorff, not collectionwise normal space, General Topology Appl. 6 (1976), 57-64.

2. C. Navy, Nonparacompactness in Paralindelof spaces, Thesis, University of Wisconsin-Madison, 1981.

3. R. Telgarsky, Total paracompactness and dispersed spaces, Bull. Acad. Polon. Sci. 16 (1968) 561-572.

4. W. S. Watson, Separation and coding (to appear).

Department of Mathematics, York University, North York, Ontario, CANADA M3J 1P3 